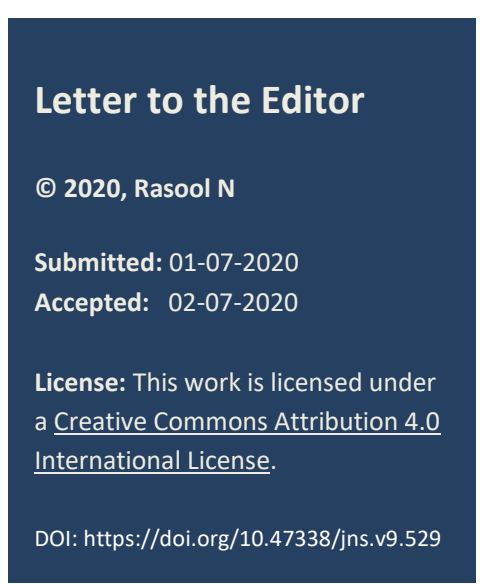

\title{
Anorectal malformations in newborn females
}

Naima Rasool*

Professor Pediatric Surgery, Ruth Pfau Medical College, PAF Base Faisal Karachi

Correspondence*: Dr. Naima Rasool, Professor Pediatric Surgery, Ruth Pfau Medical College, PAF Base Faisal Karachi, Pakistan, E-mail: naimamughal@gmail.com

\section{Dear Editor}

Anorectal malformation (ARM) in female babies is a spectrum of defects involving distal rectum, anus and urogenital tract, which manifests as absence of anal opening with or without fistulous tract with genitourinary system. Various types of anomalies range from membrane covered anus to undifferentiated cloacal malformations posing significant challenges to treating surgeons.

Due to the wide variety of anatomical variations of ARM in female, the presentation is also varied. Prompt diagnosis and plan of management is necessary to avoid complication and complexity of clinical features. The clinical presentation ranges from anatomical absence of anal opening in otherwise normal newborn baby to intestinal obstruction and sepsis with stenotic and malpositioned anal opening. Clinical history and examination are important to detect presence or absence of fistula, level of fistula in relation to pelvic muscle floor and associated anomalies. The common associations are urogenital followed by cardiac and musculoskeletal anomalies. In urogenital anomalies, vesicoureteric reflex, renal agenesis, vaginal atresia and ectopic ureter, are reported to be about $20-50 \%$ in literature.[1]

Vestibular fistula being the commonest variety (3 openings in the vestibule) and anteriorly placed anus, stenotic or membranous covered anus can be easily picked up on perineal examination. Single vestibular opening is indicative of cloaca, followed by rare varieties like rectovaginal fistula and ARM without fistula.[2] If fistula is visible on clinical examination, $\mathrm{x}-$ ray abdomen is performed to rule out pouch colon, $\mathrm{x}-$ ray spine to exclude commonly associated spinal anomalies, echocardiography for congenital heart anomalies and ultrasound of urinary tract is recommended to exclude urogenital anomalies. X-ray lateral decubitus is only required in case no fistula is visible. Ultrasound is required to detect urinary anomalies. MRI is required to de-lineate the musculature of pelvic floor. Cloacogram and or cystovaginoscopy is required in babies with single vestibular/ cloacal opening to plan early primary repair or staged procedure in cases of long common channels. MRI and endoscopy both are usually done after neonatal age. [3]

Babies without perineal fistula must be categorized as low or high, anoplasty/limited PSARP (posterior sagittal anorectoplasty) and pelvic colostomy are the immediate requirements, respectively.

Historically several techniques are reported in literature to treat anovestibular fistula including cut-back anoplasty, anal transposition, and limited PSARP, while rectovestibular fistula was treated by staged PSARP as suggested by Devries and Pena. In RVF, perineal fistula, and anteriorly placed anus, single staged procedure as ASARP (anterior sagittal anorectoplasty) is debatable, though avoiding the multiple producers and complication of stoma with equally acceptable short and long term results as reported by Okada et al. in 1992.[4] Studies have suggested good results in single staged procedure even in neonates.[5] However, operating in newborn period carries increased morbidity/mortality due to associated anomalies and anesthesia associated risks especially in developing countries. Fistula dilatation is a reasonable option for the interim period to keep adequate evacuation.[6] ASARP can be performed safely later.

On the other extreme of spectrum, cloacal malformations are found to be more complex, which needs more comprehensive evaluation and delineation of anatomy before embarking on surgery. Only diversion surgery is offered in neonatal period in addition to drainage of hydrocolpos, if present. About $30 \%$ of high cloaca may need laparoscopic assisted technically de- 
manding maneuvers which are not much feasible in neonates. [7]

Long term cosmetic and physiological results de-pend upon the recognition of correct anatomy, choice of appropriate surgical procedure and associated urogenital anomalies irrespective of age at the time of definitive surgery.[7] Keeping the patient safety paramount in the COVID awareness era, the choice of elective surgical procedure for ARM in female also depends upon the

\section{REFERENCES}

1. Stoll C, Alembik Y, Dott B, Roth MP. Associated malformations in patients with anorectal anomalies. Eur J Med Genet. 2007; 50:281-90.

2. Aldeiri B, Johal NS, De Coppi P. Meconium is not enough: look for the hole! BMJ Case Rep. 2012; 2012:bcr2012007456.

3. Bhatnagar S. Anorectal malformations (part 2). J Neonatal Surg. 2015; 4:25.

4. Okada A, Shinkichi K, Imura K. Anterior sagittal anorectoplasty for rectovestibular and anovestibular fistula. J Pediatr Surg. 1992; 27:85-8. corresponding available health care facilities and guideline in local context of developing world.

Acknowledgements: $\mathrm{Nil}$

Conflict of Interest: None declared

Source of Support: Nil

Consent to Publication: No clinical figure is used in this manuscript.

Author Contributions: Author(s) declared to fulfil authorship criteria as devised by ICMJE and approved the final version.

5. Abdul Aziz DA, Velayutham R, Osman M, Abdul Latiff $Z$, Lim FSK, Mohd Nor M. Anorectal anomaly with rectovestibular fistula: a historical comparison of neonatal anterior sagittal anorectoplasty without covering colostomy and postoperative anal dilatation to the classical three-stage posterior sagittal anorectoplasty. Open Access Surgery. 2017; 10:33-44.

6. Zamir N, Rasool N. The early outcome of primary anterior sagittal approach for low anorectal malformations in female patients. Pak J Med Sci. 2020; 36:456-60.

7. Bischoff A, Martinez-Leo B, Peña A. Laparoscopic approach in the management of anorectal malformations. Pediatr Surg Int. 2015; 31:431-7. 